\title{
Progestin therapy for endometrial cancer: The potential of fourth-generation progestin (Review)
}

\author{
KOUJI BANNO, IORI KISU, MEGUMI YANOKURA, KOSUKE TSUJI, KENTA MASUDA, ARISA UEKI, \\ YUSUKE KOBAYASHI, WATARU YAMAGAMI, HIROYUKI NOMURA, NOBUYUKI SUSUMU and DAISUKE AOKI \\ Department of Obstetrics and Gynecology, School of Medicine, Keio University, Tokyo, Japan
}

Received November 21, 2011; Accepted January 16, 2012

DOI: $10.3892 /$ ijo.2012.1384

\begin{abstract}
Progestin preparations are made of synthetic progesterone and have often been used for hormone therapy in gynecological patients with endometriosis or endometrial cancer. Hormone therapy using progestin is considered to be one of the effective means of treatment particularly when dealing with endometrial cancer (an estrogen-dependent tumor). Numerous reports have been published concerning its efficacy in advanced or recurrent cases of atypical endometrial hyperplasia or endometrial cancer. Dienogest has been developed as a fourth-generation progestin for hormone therapy for endometriosis that can be used with high safety for long periods of time. In Japan, dienogest has been recommended as a first-line drug for endometriosis-associated pain. However, its antitumor activity has also been attracting close attention following a report that this drug suppressed the proliferation in vitro of endometrial cancer-derived cell lines which failed to respond to other progestins such as medroxyprogesterine acetate (MPA). The mechanism for antitumor activity of dienogest is considered to differ from the mechanism for antitumor activity of conventional progestin preparations used for treatment of endometrial cancer. This drug is expected to be clinically applicable as a new drug for the treatment of endometrial cancer.
\end{abstract}

\section{Contents}

1. Introduction

2. Progestin therapy for endometriosis

3. Dienogest, a new progestin

4. Progestin therapy for endometrial cancer

5. Applicability of dienogest to treatment of endometrial cancer

Correspondence to: Dr Kouji Banno, Department of Obstetrics and Gynecology, School of Medicine, Keio University, 35 Shinanomachi, Shinjuku-ku, Tokyo 160-8582, Japan

E-mail:kbanno@sc.itc.keio.ac.jp

Key words: progestin, endometrial cancer, dienogest, medroxyprogesterone acetate, progesterone receptor
6. Effects of oral contraceptives and intrauterine contraceptive devices on endometrial cancer

7. Conclusion

\section{Introduction}

Synthetic progestin was first used for treatment of endometriosis in 1958 by Kistner who applied it as a false pregnancy therapy. Later, oral contraceptives (OCs) replaced synthetic progestin. In the 1980s, however, false pregnancy therapy with new drugs such as gonadotropin releasing hormone agonist (GnRHa) and danazol resulted in higher response rates than earlier methods of false pregnancy therapy and these new drugs became predominant.

However, since GnRHa therapy induces adverse reactions such as osteoporosis and climacteric symptoms associated with estrogen deficiency and because danazol induces adverse reactions such as thrombosis and masculinization, the period of continued treatment with these drugs was limited to 6 months at maximum. Under such circumstances, efforts were made to develop a new progestin having more potent progesterone activity and less androgen activity capable of replacing GnRHa and danazol for which prolonged use was difficult. As a consequence, dienogest, which has high progesterone activity and possesses anti-androgen activity, was developed. Its clinical use in Japan was approved in 2008. This drug now plays an important role in long-term management of endometriosis.

This report will describe the mechanism for the effects of progestin on endometriosis and endometrial cancer and discuss the potential of dienogest as a new progestin for the treatment of endometrial cancer.

\section{Progestin therapy for endometriosis}

Endometriosis is an estrogen-dependent disease, involving growth of endometrial tissue dependent on the blood estradiol level which rises during the menstruation cycle. This disease advances while inducing inflammation and intense pain during each menstruation period. Progestin provides a valid means of treating this disease in cases where high-dose estrogen (used for false pregnancy therapy) is not indicated and cases with estrogen-dependent tumor or thrombotic tendency. Progestin antagonizes endometrial tissue growth by suppressing the secretion of gonadotropin from the pituitary gland and the formation 
of hormones in the ovaries as well we through inducing changes in the decidual of the endometrium and subsequent endometrial atrophy. The effect of progestin in suppressing endometrial growth is comparable to that of GnRHa and danazol. The cost of progestin is lower and the incidence of estrogen deficiencyassociated adverse reactions (reduction in bone mineral density, climacteric symptoms and so on) is lower with progestin than with GnRHa and danazol. Because of these features, some investigators consider progestin to be more useful than GnRHa and danazol as a means of treating endometriosis (1). A major adverse reaction to progestin therapy is irregular bleeding, a symptom seen in about $40 \%$ of patients treated with progestin at high-dose levels. In cases of estrogen-dependent tumor and cases without thrombotic tendency, this symptom can be alleviated by oral low-dose estrogen treatment.

Medroxyprogesterone acetate (MPA) is one of the progestins most deeply studied. It has been shown that lesions diminished or disappeared, accompanied by alleviation of symptoms, in all of the 35 patients with endometriosis complaining of pain following 90-day oral treatment with this drug $(30 \mathrm{mg} /$ day $)$ (2). In a study involving oral treatment with megestrol acetate (40 mg/day) in 29 patients with endometriosis, $86 \%$ of the patients showed alleviation of symptoms such as pain and irregular bleeding (3). In 2001, a report was published, demonstrating that in patients complaining of endometriosis-associated pain, the use of an intrauterine device releasing levonorgestrel (a progesterone) (LNG-IUD) reduced menorrhea and pain during intercourse and diminished the nodular induration of Douglas' pouch (4). In the same year, strong pain-relieving effects of gestrinone (a progestin having agonist activity on androgen receptor) as compared to GnRHa were also reported.

Furthermore, Sacco et al reported the efficacy of progestin therapy on prostaglandin $\mathrm{E}_{2}\left(\mathrm{PGE}_{2}\right)$ involved in endometriosis (5). According to their report, $\mathrm{PGE}_{2}$ is produced in macrophages and ectopic endometrium in patients with endometriosis, causing up-regulation within the peritoneal cavity and increasing estrogen synthesis through up-regulation of steroidogenic acute regulatory protein (StAR) and aromatase. $\mathrm{PGE}_{2}$ additionally causes up-regulation of fibroblast growth factor-9 (FGF-9), which suppresses cell apoptosis and stimulates cell proliferation. The $\mathrm{PGE}_{2}$-stimulated estrogen synthesis leads to up-regulation of vascular endothelial growth factor (VEGF), and $\mathrm{PGE}_{2}$ itself causes up-regulation of VEGF and fibroblast growth factor-2 (FGF-2), resulting in stimulation of vascularization. Estrogen, VEGF and FGF-2 all cause positive feedback of $\mathrm{PGE}_{2}$. Dienogest is a synthetic progestin with a target set at expression of genes involved in biosynthesis of PGs (e.g., $\mathrm{PGE}_{2}$ ), as described later in detail, and is considered to exert effects against endometriosis through suppressing the $\mathrm{PGE}_{2}$-associated cascade (Fig. 1).

In patients with endometriosis, progestin therapy alleviates symptoms and diminishes lesions through suppressing endometrial growth via the diverse mechanisms mentioned above. Now, active research is under way on new preparations of progestin and new methods for clinical application of progestin.

\section{Dienogest, a new progestin}

Dienogest is a fourth-generation progestin developed as a new 19-nortestosterone derivative with higher progesterone activity and anti-androgen activity than ordinary progesterone

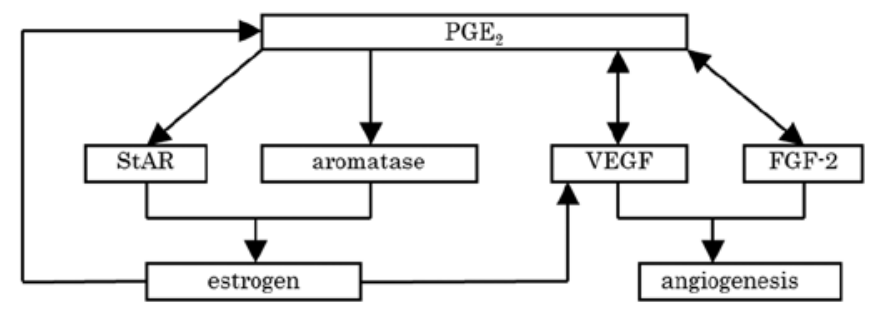

Figure 1. Effect of $\mathrm{PGE}_{2}$ on estrogen synthesis and vascularization.

preparations on endometriosis (Fig. 2). Dienogest stimulates the differentiation of endometrial stromal cells and suppresses their proliferation (6). It can be additionally characterized by no suppression of basal levels of luteinizing hormone (LH) and follicle stimulating hormone (FSH) (6). Progestin reduces the basal LH and FSH levels and thus suppresses ovarian function, while dienogest suppresses the proliferation of endometriosis cells through reducing blood estradiol level by induction of closure of dominant follicles without suppressing FSH. Furthermore, dienogest also suppresses cell proliferation through inducing G0/ G1 arrest suppressing uptake of deoxyuridine bromide (BrdU) into endometriosis cells at concentration levels $>10^{-7} \mathrm{M}$ (7). At a level of $10^{-7} \mathrm{M}$, dienogest also suppresses the TNF $\alpha$-stimulated formation of IL-8 by endometriosis cells (8). Thus, dienogest suppresses growth and inflammation of lesions. Dienogest is therefore expected to reduce pain and induce diminishment and atrophy of lesions in patients with endometriosis through suppression of ovarian function and direct suppression of endometriosis cell proliferation, with its effect in inducing atrophy of lesions having been reported to be comparable to that of GnRHa and danazol $(9,10)$. Dienogest is superior to GnRHa and danazol in terms of lack of restrictions on dosing period, milder adverse reactions and lower risk for thrombosis, thus providing a valid means of treating endometriosis which required long-term control of menstruation. Six-month treatment with dienogest has been shown to markedly alleviate dysmenorrhea, pre-menstruation pain, coital pain and extensive pelvic pain (11).

In Japan, the Guidelines on Obstetric and Gynecological Management (2011), prepared by the Japan Society of Obstetrics and Gynecology list low-dose EP combined preparations and dienogest as first-line drugs in cases of endometriosis-associated pain poorly responding to existing analgesic agents or requiring treatment of endometriosis itself. A major adverse reaction to dienogest is irregular genital bleeding ( $60 \%)$, and other adverse reactions include headache, nausea, breast discomfort, dizziness, hotflashes, reduced hemoglobin and weight gain (10).

\section{Progestin therapy for endometrial cancer}

Endometrial cancer is a malignant epithelial tumor which develops in the endometrium of uterine. It often develops in environments where estrogen secretion outweighs progesterone secretion. Endometrial cancer is treated with more diverse methods than are other malignant tumors affecting the intrapelvic organs. Total hysterectomy is the standard treatment for endometrial cancer. In inoperable cases, radiotherapy, chemotherapy and hormone therapy are applied, and combinations of these therapies are used in advanced or recurrent cases of endo- 


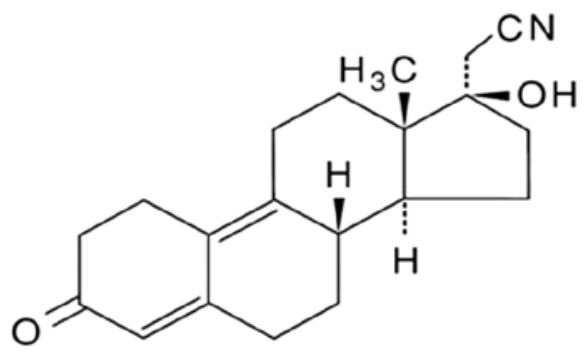

17-hydroxy-3-oxo-19-nor-17 $\alpha$-pregna-4,9-diene-21-nitrile(INN:Dienogest)

Figure 2. Chemical structure of dienogest.

metrial cancer. Of these therapies, hormone therapy is indicated in patients who wish to remain fertile after pre-menopausal early development of the cancer, patients having serious clinical problems not indicated for surgery, and patients requiring postoperative hormone replacement therapy.

Progesterone suppresses the growth of endometrial cancer, and its various derivatives have been used for hormone therapy. At present, megestrol acetate and MPA are the most frequently used progesterone preparations. Regarding the mechanism for suppressed growth of endometrial cancer, progestin was previously considered to exert this effect through down-regulation of estrogen receptors (ER), and activation of enzymes involved in estrogen metabolism. At present, the effect of progestin is known to involve cell cycle regulation by cyclin-dependent kinase (Cdk) and anti-oncogene is an important factor. Cyclin is a substance expressed during the cell cycle and is known to form a complex with specific Cdk and to advance the cell cycle. On the other hand, the products of anti-oncogenes (such as p53) and Cdk inhibitors antagonize the function of cyclin and Cdk, resulting in suppression of cell proliferation. In the glandular epithelium of endometrial tissue, several cell cycle promoting factors are expressed during the growth phase and p27 (a cyclin E-Cdk 2 complex inhibitor) is expressed during the secretory phase. Progestin is considered to reinforce $p 27$ expression, resulting in inhibition of cyclin E-Cdk2 function and suppression of the cell cycle (12).

In cases of atypical endometrial hyperplasia and endometrial cancer (particularly well-differentiated endometrioid type), progesterone receptor (PR) is often expressed, resulting in regulation of growth by progestin. The response rate to MPA is generally higher with PR-positive cancer than with PR-negative cancer, suggesting that the effect of progestin is mediated by PR (13). 17 $\beta$-hydroxysteroid dehydrogenase type 2 (17-HSD type 2 ) is an enzyme constituting the androgen and estrogen synthesis and catalyzes the conversion of E2 (potent estrogen) into E1 (inactive form). 17-HSD type 2 and PR (particular the isotype called PRB) are attracting close attention as factors enabling prediction of responses of endometrioid type endometriosis to progestin therapy (14). 17-HSD type 2 is detected in the glandular cell cytoplasm only during the endometrial secretory phase and is not detected during the proliferative phase. In endometrial tissue epithelial cells, progestin stimulates the expression of 17-HSD type 2 (15). Progestin in endometrial tissue has the possibility of exerting anti-estrogen activity in the endometrium through inducing 17-HSD type 2 and stimulating the remission of endometrial hyperplastic disease.
Various results have been reported concerning the effects of progestin used as postoperative adjuvant therapy for endometrial cancer. Urbanski et al reported that the 5-year survival rate of patients with endometrial cancer was significantly higher following postoperative adjuvant therapy with progesterone $(97 \%, \mathrm{n}=100)$ as compared to the control group $(68.6 \%, \mathrm{n}=105)$ (16). However, McDonald et al (17) and Vergote et al (18) reported that progesterone therapy failed to improve the survival rate sufficiently, and De Palo et al (19) also reported the lack of survival rate improvement following progesterone treatment, adding that postoperative adjuvant therapy is not effective and hence is not recommendable. Thus, there is no definite conclusion as to the usefulness of postoperative adjuvant therapy as a means of preventing recurrence of endometrial cancer (20).

Regarding progestin therapy for advanced or recurrent endometrial cancer, treatment of 331 cases with MPA $50 \mathrm{mg}$ three times daily resulted in complete remission in 32 cases (9.6\%) and partial remission in 26 cases $(7.9 \%)$, with the median exacerbation-free survival period being 4.0 months and the median overall survival period being 10.5 months (21). When MPA 200 or $1,000 \mathrm{mg}$ /day was administered to 229 cases of advanced or recurrent endometrial cancer, the low-dose group (200 mg/day group) showed better outcomes (17\% complete remission and $8 \%$ partial remission) than in the high-dose group (1,000 mg/day group) (9\% complete remission and 6\% partial remission) (22). Median exacerbation-free survival period also differed significantly between the low-dose group (3.2 months) and the high-dose group (2.5 months) (22). When progestin therapy is actually applied to clinical cases, high-dose oral MPA is used. Regarding its dose level, the Gynecologic Oncology Group (GOG) reported that when patients with advanced or recurrent cancer were assigned at random to the low-dose group (MPA $200 \mathrm{mg} / \mathrm{day}, \mathrm{n}=145)$ and the high-dose group (1,000 mg/ day, $n=154)$, the response rate was higher in the low-dose group ( $25 \%$, including 25 cases with complete remission and 11 with partial remission) than in the high-dose group (16\%, 14 cases with complete remission and 10 with partial remission), with the responses being particularly favorable in G1 and PR-positive cases.

Thrombosis is a serious adverse reaction to MAP. It is caused by the MPA's inhibitory activity against plasminogen activator (PA) (24). Because this adverse reaction can be fatal through inducing cerebral infarction, myocardial infarction, and pulmonary embolism, monthly checks of the clotting system are needed, and treatment with MAP needs to be discontinued upon detection of abnormality in the clotting system. Cancer tissue susceptibility to MPA is important in avoiding adverse reactions to MPA. It has been shown that susceptibility of endometrial cancer to MPA varies among individual cases, with the responses more likely to appear in cases of well-differentiated endometrial cancer and less likely in cases of poorly-differentiated endometrial cancer. Indicators available for evaluation of the susceptibility of endometrial cancer to MPA include induction of estradiol dehydrogenase, suppression of ER expression, PR expression level and atrophic changes of vagina smear.

In developed countries, early stage endometrial cancer and atypical endometrial hyperplasia have recently been increasing also among young females aged below 40 years. When dealing with young women who desire to have children, fertility-preserving treatment instead of hysterectomy is desi- 
Table I. Progestin therapy for endometrial cancer.

\begin{tabular}{lcccr}
\hline & \multicolumn{2}{c}{$\begin{array}{c}\text { Successful response } \\
\text { rate (\%) }\end{array}$} & \multicolumn{2}{c}{$\begin{array}{c}\text { Recurrence } \\
\text { rate }(\%)\end{array}$} \\
\hline Kim et al (26) & $4 / 7$ & 57 & $2 / 4$ & 50 \\
Randall and Kurman (25) & $9 / 12$ & 75 & $1 / 9$ & 11 \\
Kaku et al (27) & $9 / 12$ & 75 & $2 / 9$ & 22 \\
Imai et al (28) & $8 / 15$ & 53 & $3 / 8$ & 38 \\
Utsunomiya et al (14) & $11 / 16$ & 69 & $\mathrm{ND}$ & ND \\
Ramirez et al (37) & $62 / 79$ & 76 & $15 / 62$ & 24 \\
Niwa et al (29) & $12 / 12$ & 100 & $8 / 12$ & 67 \\
Yahata et al (30) & $7 / 8$ & 88 & $7 / 7$ & 100 \\
Ushijima et al (31) & $14 / 22$ & 64 & $8 / 14$ & 57 \\
Yamazawa et al (32) & $8 / 9$ & 89 & $2 / 8$ & 25 \\
Minaguchi et al (33) & $15 / 19$ & 79 & $5 / 15$ & 33 \\
Kamoi et al (34) & $5 / 7$ & 71 & ND & ND \\
\hline
\end{tabular}

ND, not done.

rable and progestin therapy is used for such cases. In Japan, MPA is considered to be indicated in patients satisfying all of the following five criteria: i) FIGO 1988 surgical stage Ia, ii), well-differentiated (G1) endometrioid adenocarcinoma, iii) not contraindicated for MPA therapy, iv) nulliparous woman aged below 40, and v) informed consent on the indications and limitations of MPA therapy. To summarize the domestic and overseas reports on progestin therapy applied as fertility-preserving therapy in 1997 through 2008, the lesions disappeared in 164 (75\%) of the 218 cases of endometrial cancer (corresponding to G1 endometrioid adenocarcinoma, FIGO 1988 surgical stage Ia) in response to progestin therapy. Among these 164 cases showing disappearance of lesions, recurrence was later noted in $53(36 \%)$ of the 148 cases where information on the presence/absence of recurrence was available (Table I) (14,25-34). When progestin therapy was applied to 47 cases of atypical endometrial hyperplasia, 42 cases (89\%) showed disappearance of lesions, and $8(19 \%)$ of these 42 cases later showed recurrence (Table II) $(26,27,31,35)$. Regarding the efficacy of progestin therapy on endometrial cancer, Montz et al reported that when progestin therapy was administered to cases of G1 endometrial cancer (FIGO 1988 surgical stage Ia) not indicated for surgery for reasons of influence of complications or preservation of fertility, the tumor was histologically rated as negative in 7 of the 11 cases at 6 months and 6 of the 8 cases at 12 months after the start of treatment (36). Ramirez et al applied progestin therapy to 81 cases of endomtrioid adenocarcinoma G1 and reported the outcome (37). According to their report, $76 \%$ of these cases responded to treatment, with the median time until responses being 12 weeks. Of these respondents, $24 \%$ developed recurrence, with the median time until recurrence being 19 months. Twenty patients became pregnant after completion of treatment, and no death from endometrial cancer was recorded. Thus, when progestin therapy is applied as a fertility-preserving therapy, the probability for disappearance of lesions is high but the risk for recurrence is not low, and pregnancy cannot always be achieved even after disappearance of lesions.
Table II. Progestin therapy for atypical endometrial hyperplasia.

\begin{tabular}{|c|c|c|c|c|}
\hline \multirow{2}{*}{$\begin{array}{l}\text { Author/(refs.) } \\
\text { Randall and Kurman (25) }\end{array}$} & \multicolumn{2}{|c|}{$\begin{array}{c}\text { Successful response } \\
\text { rate }(\%)\end{array}$} & \multicolumn{2}{|c|}{$\begin{array}{c}\text { Recurrence } \\
\text { rate }(\%)\end{array}$} \\
\hline & $16 / 17$ & $94 \%$ & $2 / 16$ & $13 \%$ \\
\hline Kaku et al (27) & $15 / 18$ & $83 \%$ & $2 / 15$ & $13 \%$ \\
\hline Jobo et al (27) & $11 / 12$ & $92 \%$ & $4 / 11$ & $36 \%$ \\
\hline
\end{tabular}

Treatment with progestin is particularly effective in some patients with endometrial cancer in whom PR expression has been maintained. However, PR expression is absent in many cases of endometrial cancer, thus limiting the effects of progestin on cancer. For this reason, studies on the method for restoration of PR expression in endometrial cancer have been conducted at the gene and protein levels. To put it concretely, several preclinical studies have been carried out on the control of epigenetic mutations (extensive hypomethylation or hypermethylation of anti-oncogenes, and so on) often seen in cases of endometrial cancer for the purpose of stimulating apoptosis and restoration of susceptibility of cancer to chemotherapy (38). In cases of cancer where transcription of anti-oncogenes is usually regulated by DNA methylation, DNA methyltransferase inhibitors (DNMTi) stimulate the re-expression of anti-oncogenes, contrary to their usual action. Furthermore, since histone deacetylation maintains transcription silencing in the presence of cancer, histone deacetylase inhibitors (HDACi) stimulate transcription. Combined use of these two inhibitors is often more effective than separate use of one of them. One possible mechanism for the augmented efficacy of this combination is that HDACi directly reduces the activity of DNMT3B through weakening the stability of mRNA of DNMT3B, a kind of DNMT $(39,40)$. The second possible mechanism is involvement of HDAC with a methylated DNA sequence. However, it has not yet been fully clarified how the cross-talk between these two enzyme groups occurs and how DNMT1/HDACi therapy exerts therapeutic efficacy (41). Uncombined treatment with DNMTi or HDACi or treatment with their combination can restore the expression of anti-oncogenes in endometrial cell lines, leading to less cell proliferation and activation of apoptosis (42). This therapeutic strategy has been shown in several studies to stimulate up-regulation of PR in endometrial cancer cell lines. PRB, a kind of PR, is found in a hypermethylated form in $75 \%$ of endometrical cancer, and its expression was noted following treatment of endometrical cell lines with decitabine (a kind of DNMTi) in a preclinical study (43). This finding suggests that the use of epigenetic regulatory factors can also stimulate PR expression and restoration of long-lasting susceptibility to progestin in vivo. Furthermore, regarding PR hypermethylation which is one of the mechanisms responsible for disappearance of PR in endometrial cancer, some studies revealed that treatment with DNMTi alone or DNMTi + HDACi stimulated re-expression of PR at both the mRNA level and the protein level $(39,44)$.

Many patients with endometrial cancer show no response or are resistant to progestin therapy, but the molecular mechanism for the lack of response or development of resistance to progestin has not been fully elucidated. Epidermal growth factor receptor (EGFR) is estimated as a factor involved in these cases. 
EGFR was detected in histological specimens and cell lines of endometrial cancer and is known to be excessively expressed in endometrial cancer, although its role in resistance to progestin has not been clarified. Ai et al analyzed differences in EGFR function and resistance to progestin in relation to presence/ absence of PRB expression in endometrial cancer, reporting that EGFR was detected in $60 \%$ of PRB-positive specimens and $90.5 \%$ of PRB-negative specimens. Furthermore, although EGFR expression was higher in progestin-resistant KLE cells than in progestin-susceptible Ishikawa cells, PRB expression level was higher in Ishikawa cells than in KLE cells. When further EGFR expression was stimulated in Ishikawa cells, the susceptibility to progestin decreased, accompanied by reduction in PRB expression. AG1478 (a specific inhibitor for EGFR tyrosine kinase) effectively suppressed the proliferation of EGFR-overexpressed endometrial cancer cells (45). On the basis of these findings, we may say that excessive expression of EGFR in endometrial cancer cells can reduce the susceptibility to progestin therapy. Therefore, inhibitors specific to EGFR tyrosine kinase are likely to be effective against endometrial cancer resistant to progestin therapy.

\section{Applicability of dienogest to treatment of endometrial cancer}

The fourth-generation progestin dienogest is an effective means of treating endometriosis. In Japan, it has been recommended as a first-line drug to be selected prior to treatment with GnRHa or danazol. At present, its anti-tumor activity is also attracting close attention. Because of high progesterone activity, dienogest has been actively studied in regards to its effects on hormonedependent tumors among other aspects.

Regarding the effects of dienogest on endometrial cancer, Katsuki et al evaluated its effects in vivo using mice in which a subcutaneous tumor had been induced by two cells lines derived from endometrial cancer (46). The two cell lines of endometrial cells used in their study were HEC-88nu cell (expressing ER and but expressing PR) and Ishikawa cells (expressing both ER and PR). Mice with subcutaneous tumors induced by these cell lines were orally treated with dienogest (0.01-1.0 mg/kg/day) or MPA (100 mg/kg/day). The mice with tumors induced by HEC-88nu cells did not respond to MPA but showed suppressed tumor growth in response to dienogest. The tumors induced by Ishikawa cells showed suppressed growth in vivo in response to both dienogest and MPA, with the lowest dose level exhibiting a suppressive activity considerably lower with dienogest than with MPA. Although the susceptibility of endometrial cancer to MPA is known to correlate with the PR expression level, dienogest suppressed the proliferation of PR-negative HEC-88nu cells in mice.

Nakamura et al proposed inhibition of neovascularization as a mechanism for cell proliferation suppression by dienogest, reporting that vascularization in the mouse tissue implanted with S-180 tumor cells was markedly suppressed by 5-day consecutive oral treatment with dienogest ( $1 \mathrm{mg} / \mathrm{kg} /$ day) (47). Neovascularization is an important step for tumor cell growth, invasion and metastasis in lesions of cancer in general (including endometrial cancer), and its inhibition can lead to suppression of cancer cell proliferation. Dienogest is also estimated to exert efficacy against endometrial cancer through suppressing neovascularization. In the cascade for neovascularization, decomposition of extracellular matrix by proteases such as PA is important. Inhibition of neovascularization can be achieved by suppression of this reaction, and MPA capable of inhibiting PA formation actually suppressed neovascularization (24). However, according to the report by Nakamura et al, MPA suppressed the formation of PA in vascular endothelial cells while dienogest did not suppress PA formation, thus suggesting that the mechanism for inhibition of neovascularization by dienogest differs from that by MPA. Although the exact mechanism for inhibition of neovascularization is not known, it is evident that the dienogest inhibits neovascularization. This feature of dienogest seems to be associated with its anti-tumor activity.

Katayama et al evaluated the effect on neovascularization using a rat model of endometrial implantation, demonstrating that oral dienogest treatment $(1 \mathrm{mg} / \mathrm{kg})$ suppressed formation of new small vessels, accompanied by decrease in perivascular $\alpha$ smooth myofibril actin level in implanted endometrium, suggesting structural changes of small vessels possibly associated with the drug's effect in suppressing neovascularization (48).

Dienogest exerts suppression of cell cycle in addition to inhibition of neovascularization. Cdk and anti-oncogenes play an important role in regulation of cell cycle, and progestin therapy suppresses the cell cycle through reinforcing the expression of p27 (a cyclin E-Cdk2 inhibitor). Shimizu et al reported that dienogest suppressed the expression of cyclin D1 gene and this inhibited the proliferation of endometrial epithelial cell line (12). According to their report, the epithelial cells showed no change in expression of anti-oncogenes such as $p 27$ and $p 53$ even after treatment with dienogest and that only the expression of cyclin D1 mRNA and cyclin E1 mRNA decreased in response to dienogest treatment, thus indicating that dienogest directly suppresses endometrial epithelial cells.

Dienogest is closely associated with $\mathrm{PGE}_{2}$. $\mathrm{PGE}_{2}$ serves as an important mediator in maintaining the homeostasis involved in cell proliferation, apoptosis, migration, infiltration, and neovascularization. $\mathrm{PGE}_{2}$ synthases include $\mathrm{COX}-2$ and microsomal $\mathrm{PGE}_{2}$ synthase-1 (mPGES-1). These enzymes are produced by various inflammatory stimuli and their formation is regulated by NF- $\kappa$ B. COX-1 and mPGES-1 are abundantly expressed in endometrial epithelial cells and epithelial cancer tissue, suggesting that $\mathrm{PGE}_{2}$ serves as a major mediator involved in pathophysiology and pathogenesis of gynecologic diseases accompanied by abnormal growth or inflammation of endometrium. Shimizu et al, mentioned above, evaluated the influence of dienogest on $\mathrm{PGE}_{2}$ formation, reporting that dienogest inhibited $\mathrm{PGE}_{2}$ formation and this inhibitory action was lifted by PR antagonist RU-486 (49). In that report, dienogest was shown to inhibit the expression of $\mathrm{PGE}_{2}$ synthase mRNA and protein as well as NF- $\kappa \mathrm{B}$ activity, with such activity remaining to be seen $24 \mathrm{~h}$ after treatment. If these findings are combined with the selective agonist activity of dienogest to $\mathrm{PR}$, it seems likely that activation of $\mathrm{PR}$ reduces the expression of COX-2 and mPGES-1.

Considering the findings that dienogest suppressed the endometrial cancer-derived cell lines having failed to respond to MPA and that it suppressed neovascularization and the cell cycle and inhibited $\mathrm{PGE}_{2}$ formation through selective antagonist activity to PR, this drug is very likely to manifest anti-tumor activity in endometrial cancer and is promising as a candidate drug for clinical application. 


\section{Effects of oral contraceptives and intrauterine contra- ceptive devices on endometrial cancer}

Endometrial cancer is known as one of the gynecologic cancers whose risk can be reduced by intake of oral contraceptives (OCs). Low-dose OCs contain estrogen and progestin and are known to suppress ovulation and endometrial growth and to reduce $\mathrm{PG}$ formation. The effect on endometrial cancer has been considered as one of the potential side benefit of OCs. According to the case-control study carried out in 1983 by the Centers for Disease Control and Prevention (CDC), USA, the effect of OCs on endometrial cancer was most marked in nulliparous women, with the odds ratio being $0.4(0.2-0.9)$ relative to the untreated group. In the United States, it has been reported that 2,000 women annually successfully avoid development of uterine body cancer through intake of OCs (50). Vesey and Painter recruited 17,032 women aged between 25 and 39 in 1968 through 1974 and reported the results of follow-up of 15,292 women until age 45 (51). According to their report, the relative risk (RR) for endometrial cancer was $0.1(0.0-0.7)$ for the OC intake group as compared to the untreated group, with the RR being $1.0(0.4-2.5)$ following intake of OCs for 48 months or less and $0.3(0.1-0.7)$ following intake of OCs for 97 months or more.

LNG-IUD, which has been approved in Japan as a contraceptive agent, exerts contraceptive activity through powerfully suppressing endometrial growth by the direct action of progesterone on endometrium. At present, LNG-IUD is attracting close attention because of advantages other than contraceptive efficacy. Those advantages include powerful reduction of menstrual blood volume through suppression of endometrial growth, accompanying relief of menstrual pain, endometrium protective effective and so on. Among others, it has been shown that the use of LNG-IUD in combination with hormone replacement therapy during or after menopause (even hormone replacement therapy using estrogen alone) can prevent endometrial cancer. Its efficacy in suppressing the growth of endometrium has also been shown in patients with non-atypical endometrial hyperplasia, with the responses seen in $92 \%$ of all cases within 2 years after insertion and adequate responses within one year after insert in $96 \%$ of these respondents (52).

\section{Conclusion}

Following the westernization of lifestyle in Japan, the prevalence of obesity, diabetes mellitus and hypertension has risen among Japanese people, accompanied by a recent trend for increase in the prevalence of endometrial cancer. The number of patients with endometrial cancer will further increase from now on under the influence of additional factors such as low birth rate, elevation at the age of first child delivery and so on. Under such circumstances, studies on the efficacy of dienogest on endometrial cancer may lead to advances in the treatment of endometrial cancer and are therefore valuable.

So that anti-tumor efficacy can be achieved with synthetic progesterone such as MPA, conventionally used for hormone therapy in cases of endometrial cancer, the progesterone needs to be administered at high-dose levels (in the order of several hundreds of milligrams). Furthermore, thrombosis can be induced as a serious adverse reaction to such therapy, thus requiring close monitoring of platelet count and the clotting system during this therapy and discontinuation of the therapy upon appearance of abnormalities. Even when platelet count and indicators of the clotting system are within the normal range, adverse reactions to this therapy can appear, and there are restrictions on this therapy such as the necessity of avoiding administration to patients with habit-related chronic diseases (atherosclerosis, hypertension, hyperlipidemia, and obesity) or a history of thrombosis.

PR plays an important role in endometrial cancer, and the response rate of endometrial cancer to progestin therapy varies greatly depending on PR expression level. However, PR is not expressed with a high level in all cases of endometrial cancer. There are cases where PR disappears due to hypermethylation or cases where PR expression is decreased by excessive EGFR expression. In such cases, the susceptibility of endometrial cancer to progestin is lower than usual, but PR expression can recover, and it is possible to devise a new therapeutic strategy by which re-expression of PR is promoted to enable progestin therapy even in cases of PR-negative endometrial cancer failing to respond to conventional progestin therapy. Such a new strategy is expected to allow application of progestin therapy to more cases of endometrial cancer in the future.

Dienogest exerts high efficacy against PR-positive endometrial cancer, as is the case with other progestin preparations. Dienogest has several additional advantages not possessed by conventional progestins. Through the effects of dienogest, there is a possibility that endometrial cancer showing poor or no responses to conventional progestin therapies responds well to dienogest. Considering that dienogest suppressed not only the proliferation of PR-positive cancer cells but also the proliferation of PR-negative but ER-positive cancer cells, it is likely that dienogest also stimulates suppression of cell proliferation via some mechanisms involving ER. Furthermore, the mechanism for suppression of neovascularization by dienogest has not been shown to involve suppression of PA in vascular endothelial cells but has been demonstrated to involve changes in vascular structure. It is desirable to clarify the exact mechanism for suppression of neovascularization by dienogest from now on.

As illustrated above, dienogest has been shown to suppress the proliferation of endometrial cancer-derived cell lines having failed to respond to MPA in vitro, to suppress cell proliferation even at much lower concentration levels as compared to MPA, to suppress neovascularization and to inhibit the cell cycle (through suppression of cyclin D1 and E1 expression), $\mathrm{PGE}_{2}$ synthases (found abundantly in cancer tissue) and so on. These findings suggest dienogest to be promising as a new drug for treatment of endometrial cancer. When dealing with endometrial cancer, the efficacy of the current progestin therapy is lower than that of surgery, and the recurrence rate is higher with progestin therapy. Furthermore, women whose endometrial cancer has disappeared in response to progestin therapy cannot always become pregnant. With this in mind, dienogest is expected to provide a safer and more effective means of treatment requiring lower dose levels, as compared to conventional progesterone, in patients with endometrial cancer failing to respond to MPA or not indicated for existing progestin therapy because of adverse reactions (e.g., patients with thrombotic tendency or having a history of habit-related chronic disease). Further clinical studies on this drug are desirable in the future. 


\section{References}

1. Vercellini P, Cortesi I and Crosignani PG: Progestins for symptomatic endometriosis: a critical analysis of the evidence. Fertil Steril 68: 393-401, 1997.

2. Moghissi KS and Boyce CR: Management of endometriosis with oral medroxyprogesterone acetate. Obstet Gynecol 47: 265-267, 1976.

3. Schlaff WD, Dugoff L, Damewood MD and Rock JA: Megestrol acetate for treatment of endometriosis. Obstet Gynecol 75: 646-648, 1990.

4. Fedele L, Bianchi S,Zanconato G, Portuese A and Raffaelli R: Use of a levonorgestrel-releasing intrauterine device in the treatment of rectovaginal endometriosis. Fertil Steril 75: 485-488, 2001.

5. Sacco K, Portelli M, Pollacco J, Schembri-Wismayer P and Calleja-Agius J: The role of prostaglandin E2 in endometriosis. Gynecol Endocrinol 27: 1-5, 2011

6. Okada H, Nakajima T, Yoshimura T, Yasuda $\mathrm{K}$ and Kanzaki $\mathrm{H}$ The inhibitory effect of dienogest, a synthetic steroid, on the growth of human endometrial stromal cells in vitro. Mol Hum Reprod 7: 341-347, 2001

7. Fu L, Osuga Y, Morimoto C, Hirata T, Hirota Y, Yano T and Taketani Y: Dienogest inhibits BrdU uptake with G(0)/G(1) arrest in cultured endometriotic stromal cells. Fertil Steril 89: 1344-1347, 2008.

8. Horie S, Harada T, Mitsunari M, Taniguchi F, Iwabe $T$ and Terakawa N: Progesterone and progestational compounds attenuate tumor necrosis factor alpha-induced interleukin- 8 production via nuclear factor kappa B inactivation in endometriotic stromal cells. Fertil Steril 83: 1530-1535, 2005.

9. Haney AF and Weinverg JB: Reduction of the intraperitoneal inflammation associated with endometriosis by treatment with medroxyprogesterone acetate. Am J Obstet Gynecol 159: 450-454, 1988.

10. Harada T, Momoeda M, Taketani Y, Aso T, Fukunaga M, Hagino $H$ and Terakawa N: Dienogest is as effective as intranasal buserelin acetate for the relief of pain symptoms associated with endometriosis - a randomized, double-blind, multicenter, controlled trial. Fertil Steril 91: 675-681, 2009.

11. Harada T and Taniguchi F: Dienogest: a new therapeutic agent for the treatment of endometriosis. Womens Health (Lond Engl) 6: 27-35, 2010.

12. Shimizu Y, Takeuchi T, Mita S, et al: Dienogest, a synthetic progestin, inhibits the proliferation of immortalized human endometrial epithelial cells with suppression of cyclin D1 gene expression. Mol Hum Reprod 15: 693-701, 2009.

13. Ito $\mathrm{K}$, Utsunomiya $\mathrm{H}$, Yaegashi $\mathrm{N}$ and Sasano $\mathrm{H}$ : Biological roles of estrogen and progesterone in human endometrial carcinoma new developments in potential endocrine therapy for endometrial cancer. Endocr J 54: 667-679, 2007.

14. Utsunomiya H, Suzuki T, Ito K, et al: The correlation between the response to progestogen treatment and the expression of progesterone receptor $\mathrm{B}$ and 17beta-hydroxysteroid dehydrogenase type 2 in human endometrial carcinoma. Clin Endocrinol 58: 696-703, 2003.

15. Yang S, Fang Z, Gurates B, Tamaura M, Miller J, Ferrer K and Bulun SE: Stromal PRs mediate induction of 17beta-hydroxysteroid dehydrogenase type 2 expression in human endometria epithelium: a paracrine mechanism for inactivation of E2. Mol Endocrinol 15: 2093-2105, 2001.

16. Urbański K, Karolewski K, Kojs Z, Klimek M and Dyba T: Adjuvant progestagen therapy improves survival in patients with endometrial cancer after hysterectomy. Results of one-institutional prospective clinical trial. Eur J Gynaecol Oncol 15: 98-104, 1993.

17. McDonald CC, Thorogood J and Mason MK: A randomized trial of progestogens in the primary treatment of endometrial carcinoma. Br J Obstet Gynecol 95: 166-174, 1988.

18. Vergote I, Kjorstad K, Abeler V and Kolstad P: A randomized trial of adjuvant progestagen in early endometrial cancer. Cancer 64: 1011-1016, 1989

19. De Palo G, Mangioni C, Periti P, Del Vecchio M and Marubini E: Treatment of FIGO (1971) stage I endometrial carcinoma with intensive surgery, radiotherapy and hormonotherapy according to pathological prognostic groups. Long-term results of a randomised multicentre study. Eur J Cancer 29A: 1133-1140, 1993.

20. Martin-Hirsch PL, Lilford RJ and Jarvis GJ: Adjuvant progestagen therapy for the treatment of endometrial cancer: review and metaanalyses of published randomised controlled trials. Eur J Obstet Gynecol Reprod Biol 65: 201-207, 1996.
21. Thigpen JT, Blessing JA and DiSaia PJ: A randomized comparison of doxorubicin alone versus doxorubicin plus cyclophosphamide in the management of advanced or recurrent endometrial carcinoma: A Gynecologic Oncology Group study. J Clin Oncol 12: 1408-1414, 1994

22. Thigpen JT, Brady MF and Alvarez RD: Oral medroxyprogesterone acetate in the treatment of advanced or recurrent endometrial carcinoma: a dose-response study by the Gynecologic Oncology Group. J Clin Oncol 17: 1736-1744, 1999.

23. Rose PG, Brunetto VL, van Le L, Bell J, Walker JL and Lee RB: A phase II trial of anastrozole in advanced recurrent or persistent endometrial carcinoma: a Gynecologic Oncology Group study. Gynecol Oncol 78: 212-216, 2000.

24. Ashino-Fuse, H, Takano Y, Oikawa T, Shimamura M and Iwaguchi T: Medroxyprogesterone acetate, an anti-cancer and antiangiogenic steroid, inhibits the plasminogen activator in bovine endothelial cells. Int J Cancer 44: 859-864, 1989.

25. Randall TC and Kurman RJ: Progestin treatment of atypical hyperplasia and well-differentiated arcinoma of the endometrim in woman under 40. Obstet Gynecol 90:434-440, 1997.

26. Kim YB, Holscneider CH, Ghosh K, Nieberg RK and Montz FJ: Progetin alone as primary treatment of endometrial carcinoma in premenopausal woman. Report of seven cases and review of the literature. Cancer 79: 320-327, 1997.

27. Kaku T, Yoshikawa $\mathrm{H}$, Tsuda $\mathrm{H}$, et al: Conservative therapy for adenocarcinoma and atypical endometrial hyperplasia of the endometrium in young women: central pathologic review and treatment outcome. Cancer Lett 167: 39-48, 2001

28. Imai M, Jobo T, Sato R, Kawaguchi $M$ and Kuramoto $H$ : Medroxyprogesterone acetate for patients with adenocarcinoma of the endometrium who wish to presrve the uteus-usefulness and limitations. Eur J Gynaecol Oncol 22: 217-220, 2001.

29. Niwa K, Tagami K, Lian Z, Onogi K, Mori H and Tamaya T: Outcome of fertility-preserving treatment in young women with endometrial carcinomas. BJOG 112: 317-320, 2005.

30. Yahata T, Fujita K, Aoki Y and Tanaka K: Long-term conservative therapy for endometrial adenocarcinoma in young women. Hum Reprod 21: 1070-1075, 2005.

31. Ushijima K, Yahata H, Yoshikawa H, et al: Multicenter phase II study of fertility-sparing treatment with medroxyprogesterone acetate for endometrial carcinoma and atypical hyperplasia in young women. J Clin Oncol 25: 2798-2803, 2007.

32. Yamazawa K, Hirai M, Fujito A, et al: Fertility-preserving treatment with progestin, and pathological criteria to predict response, in young women with endometrial cancer. Hum Reprod 22: 1953-1958, 2007.

33. Minaguchi T, Nakagawa S, Takazawa Y, et al: Combined phosphoAkt and PTEN expressions associated with post-treatment hysterectomy after conservative progestin therapy in complex atypical hyperplasia and stage Ia, G1 adenocarcinoma of the endometrium.Cancer Lett 248: 112-122, 2006.

34. Kamoi S, Ohaki Y,Mori O, Kurose K, Fukunaga M and Takeshita T: Serial histologic observation of endometrial adenocarcinoma treated wuth high-dose progestin until complete disappearance of carcinomatous foci-review of more than 25 biopsies from five patients. Int J Gynecol Cancer 18: 1305-1314, 2008.

35. Jobo T, Kawaguchi M, Imai M and Kuramoto H: Treatment for complex atypical hyperplasia of the endometrium. Eur J Gynaecol Oncol 22: 365-368, 2001.

36. Montz FJ, Bristow RE, Bovicelli A, Tomacruz R and Kurman RJ: Intrauterine progesterone treatment of early endometrial cancer. Am J Obctet Gynecol 186: 651-657, 2002.

37. Ramirez PT, Frumovitz M, Bodurka DC, Sun CC and Levenback C Hormonal therapy for the management of grade 1 endometrial adenocarcinoma. Gynecol Oncol 95: 133-138, 2004.

38. Yang S, Thiel KW, De Geest K and Leslie KK: Endometrial cancer: reviving progesterone therapy in the molecular age. Discov Med 12: 205-212, 2011

39. Ren Y, Liu X, Ma D, Feng Y and Zhong N: Down-regulation of the progesterone receptor by the methylation of progesterone receptor gene in endometrial cancer cells. Cancer Genet Cytogenet 175: 107-116, 2007.

40. Xiong Y, Dowdy SC, Podratz KC, Jin F, Attewell JR, Eberhardt NL and Jiang SW: Histone deacetylase inhibitors decrease DNA methyltransferase-3B messenger RNA stability and down-regulate de novo DNA methyltransferase activity in human endometrial cells. Cancer Res 65: 2684-2689, 2005.

41. Kondo Y: Epigenetic cross-talk between DNA methylation and histone modifications in human cancers. Yonsei Med J 50: 455-463, 2009. 
42. Balch C, Matei DE, Huang TH-M and Nephew KP: Role of epigenomics in ovarian and endometrial cancers. Epigenomics 2: 419-447, 2010.

43. Sasaki M, Dharia A, Oh BR, Tanaka Y, Fujimoto S and Dahiya R: Progesterone receptor $\mathrm{B}$ gene inactivation and $\mathrm{CpG}$ hypermethylation in human uterine endometrial cancer. Cancer Res 61: 97-102, 2001.

44. Xiong Y, Dowdy SC, Gonzalez Bosquet J, Zhao Y, Eberhardt NL, Podratz KC and Jiang S-W: Epigenetic-mediated upregulation of progesterone receptor B gene in endometrial cancer cell lines. Gynecol Oncol 99: 135-141, 2005.

45. Ai Z, Wang J, Wang Y, Lu L, Tong J and Teng Y: Overexpressed epidermal growth factor receptor (EGFR)-induced progestin insensitivity in human endometrial carcinoma cells by the EGFR/ mitogen-activated protein kinase signaling pathway. Cancer 116: 3603-3613, 2010.

46. Katsuki Y, Shibutani Y, Aoki D and Nozawa S: Dienogest, a novel synthetic steroid, overcomes hormone-dependent cancer in a different manner than progestins. Cancer 79: 169-176, 1997.

47. Nakamura M, Katsuki Y, Shibutani Y and Oikawa T: Dienogest, a synthetic steroid, suppresses both embryonic and tumor-cellinduced angiogenesis. Eur J Pharmacol 386: 33-40, 1999.
48. Katayama H, Katayama T, Uematsu K, et al: Effect of dienogest administration on angiogenesis and hemodynamics in a rat endometrial autograft model. Hum Reprod 25: 2851-2858, 2010.

49. Shimizu Y, Mita S, Takeuchi T, Notsu T, Mizuguchi K and Kyo S: Dienogest, a synthetic progestin, inhibits prostaglandin E2 production and aromatase expression by human endometrial epithelial cells in a spheroid culture system. Steroids 76: 60-67, 2011.

50. The Centers for Diseases Control Cancer and Steroid Hormone Study: Oral contraceptive use and the risk of endometrial cancer. JAMA 249: 1600-1604, 1983.

51. Vessey MP and Painter R: Endometrial and ovarian cancer and oral contraceptives - findings in a large cohort study. Br J Cancer 71: 1340-1342, 1995

52. Varma R, Soneja H, Bhatia K, Ganesan R, Rollason T, Clark TJ and Gupta JK: The effectiveness of a levonorgestrel-releasing intrauterine system (LNG-IUS) in the treatment of endometrial hyperplasia - a long-term follow-up study. Eur J Obstet Gynecol Reprod Biol 139: 169-175, 2008. 